\title{
Value of the BI-RADS classification in MR-Mammography for diagnosis of benign and malignant breast tumors
}

\author{
Christian Sohns • Martin Scherrer • Wieland Staab • \\ Silvia Obenauer
}

Received: 16 December 2010/Revised: 10 May 2011 / Accepted: 20 May 2011 /Published online: 31 July 2011

(C) The Author(s) 2011. This article is published with open access at Springerlink.com

\begin{abstract}
Aim To assess whether the BI-RADS classification in MRMammography (MRM) can distinguish between benign and malignant lesions.

Material and method 207 MRM investigations were categorised according to BI-RADS. The results were compared to histology. All MRM studies were interpreted by two examiners. Statistical significance for the accuracy of MRM was calculated.

Results A significant correlation between specific histology and MRM-tumour-morphology could not be reported. Mass (68\%) was significant for malignancy. Significance raised with irregular shape (88\%), spiculated margin (97\%), rim enhancement (98\%), fast initial increase $(90 \%)$, post initial plateau $(65 \%)$, and intermediate T2 result $(82 \%)$. Highly significant for benignity was an oval mass $(79 \%)$, slow initial increase (94\%) and a hyperintense $\mathrm{T} 2$ result (77\%), also an inconspicuous MRM result (77\%) was often seen in benign histology. Symmetry (90\%) and further post initial increase $(90 \%)$ were significant, whereas a regional distribution (74\%) was lowly significant for benignity.

Conclusion On basis of the BI-RADS classification an objective comparability and statement of diagnosis could
\end{abstract}

C. Sohns $(\square)$

Department of Cardiology and Pneumology, Heart Center,

Georg-August-University of Göttingen,

Robert-Koch-Strasse 40,

37075 Göttingen, Germany

e-mail: csohns@med.uni-goettingen.de

M. Scherrer $\cdot$ W. Staab $\cdot$ S. Obenauer

Department of Radiology, Georg-August-University of Göttingen,

Göttingen, Germany be made highly significant. Due to the fact of falsenegative and false-positive MRM-results, histology is necessary.

Keywords Magnetic resonance imaging · Breast tumours · Breast cancer

\section{Introduction}

Breast cancer is the most common cancer in women aging more than 40 years [1]. In early studies magnetic resonance imaging of the breast (MRM) was used as a tool to further characterize suspicious clinical or mammographic examinations. However indications for MRM are high risk patients, preoperative estimation of the extent of the disease in patients with newly diagnosed breast cancer, especially lobular invasive carcinomas, patients with breast implants, cancer of unknown primary and the differentiation between scar and tumour recurrence [2-7]. Breast cancers enhance brightly compared to normal breast tissue and normal biopsy scars, which enhance little or even not at all [8]. However, MRM techniques vary across the world. In 1999 the "Lesion Diagnosis Working Group" devised the recommendations for standard MRM terms based on architectural features described by the ACR (American College of Radiology) BIRADS system (Breast Imaging Reporting and Data System) [8, 9]. Furthermore, Ikeda et al. developed and standardized the reproducibility in a lexicon for reporting contrast enhanced breast magnetic resonance imaging examinations. This lexicon adapted American College of Radiology Breast Imaging Reporting and Data System terminology for MRM reporting, including recommendations for reporting clinical history, technical parameters for MRM, descriptions for general breast 
Table 1 Distribution of the histological malignant and benign lesions

\begin{tabular}{llll}
\hline Carcinomas & Number (n) & Benign Tumors & Number (n) \\
\hline Invasive ductal carcinoma, IDC & 32 & Ductal hyperplasia, DH & 21 \\
Invasive lobular carcinoma, ILC & 18 & Fibroadenoma, FI & 18 \\
Ductal carcinoma in situ, DCIS & 17 & Fibrocystic changes, FZM & 16 \\
Tubular carcinoma, TU & 10 & Papilloma, PA & 9 \\
Lobular carcinoma in situ, LCIS & 9 & Benign not categorisable, A & 8 \\
IDC + Extensiv intraductal carcinoma, EIC & 8 & Adenosis, SA & 13 \\
Ductal and lobular carcinoma, DLC & 6 & Phyllodes Tumor, PB & 3 \\
Mucinous carcinoma, MUC & 5 & Radial scar, RN & 2 \\
Carcinosarcoma, S & 2 & Atypical Hyperplasia, AH & 2 \\
Others & 5 & Others & 3 \\
Total & 112 & Total & 95 \\
\hline
\end{tabular}

composition, morphological and kinetically characteristics of mass lesions or regions of abnormal enhancement, overall impression and management recommendations [9, 10]. Consequently, the aim of this study was to assess whether a BI-RADS associated MRM algorithm delivers a clinical significant prognosis for the diagnosis of breast tumours.

\section{Materials and methods}

\section{Study subjects}

In a consecutive series between April 2004 and January 2008, 207 female patients with histological proven breast lesions have been evaluated. Histology was predominantly reached by either preoperative wire localization in MRM or by ultrasound or stereotactic guided biopsies. In case of malignancy reference standard was the pathology after surgery. All of the patients have had a preoperative MR mammography. In addition all patients underwent a digital mammography (Senographe 2000D, GE Medical Systems). Patient details were recorded with the purpose to identify the mammograms and for accessing demographic informa- tion. From the request card, logbooks and hospital database, record was made of age, referring speciality, date of admission, and anamnesis. Written informed consent was obtained from each patient. The median age of the study group was 54 years. Due to different indications with regards to suspicious breast findings, including a palpable mass, patients with high risk, and referrals from other screening investigations like mammography or ultrasound, all patients were referred from our academic Breast Center. 112 patients had a histological proven breast cancer and 95 patients had a histological proven benign tumour (Table 1). In case of a benign lesion the mean follow up interval was 6 months.

\section{MRM technique}

All 207 MRM examinations were performed with the patient prone in a $1.5-\mathrm{T}$ commercially available system (Symphony and Sonata, Siemens, Erlangen, Germany) using a dedicated surface breast coil. The standardized imaging sequence started with a bilateral axial T2-weighted turbo spin-echo sequence (GRAPPA factor 2; 8.900/207; flip angle $90^{\circ}$, spatial resolution $0.8 \times 0.7 \times 3 \mathrm{~mm}$; acquisition time $2 \min 15 \mathrm{~s}$ ) and a bilateral turbo spin-echo inversion recovery sequence with reconstruction (GRAPPA
Table 2 Shape of the mass enhancement in histological proven malignant and benign tumors

\begin{tabular}{lllll}
\hline $\begin{array}{l}\text { Mass } \\
\text { Shape }\end{array}$ & $\begin{array}{l}\text { Malignant } \\
\text { Number (n) }\end{array}$ & $\begin{array}{l}\text { Benign } \\
\text { Number (n) }\end{array}$ & Total & p-value \\
\hline Round & 5 & 5 & 10 & 0.5196 \\
Irregular & 60 & 8 & 68 & $0.0000^{* * *}$ \\
Lobulated & 11 & 14 & 25 & 0.1927 \\
Oval & 3 & 11 & 14 & $0.0111^{*}$ \\
\hline
\end{tabular}


Table 3 Margin of the mass enhancement in histological proven malignant and benign tumors

\begin{tabular}{lllll}
\hline $\begin{array}{l}\text { Mass } \\
\text { Margin }\end{array}$ & $\begin{array}{l}\text { Malignant } \\
\text { Number (n) }\end{array}$ & $\begin{array}{l}\text { Benign } \\
\text { Number (n) }\end{array}$ & Total & p-value \\
\hline Smooth & 3 & 16 & 19 & $0.0004^{* * *}$ \\
Irregular & 47 & 20 & 67 & $0.0010^{* *}$ \\
Spiculated & 29 & 1 & 30 & $0.0000^{* * *}$ \\
\hline
\end{tabular}

factor 2; 8.420/70; inversion time $150 \mathrm{~ms}$; flip angle $180^{\circ}$, spatial resolution $1.7 \times 1.4 \times 3 \mathrm{~mm}$; acquisition time $2 \mathrm{~min}$ and $33 \mathrm{~s}$ ) were performed in identical slice positions. Afterwards an unenhanced axial T1-weighted gradient-echo sequence (Flash 2D, GRAPPA factor 2; TR/TE 113/5; flip angle $80^{\circ}$; spatial resolution $1.1 \times 0.9 \times 3 \mathrm{~mm}$; acquisition time 1 min per measurement) was performed. An intravenous bolus injection of $0.1 \mathrm{mmol} / \mathrm{kg}$ body weight gadopentetate dimeglumine (Magnevist, Bayer Schering Pharma, Berlin, Germany) was used at $3 \mathrm{ml} / \mathrm{s}$ with an automatic injector and followed immediately by $20 \mathrm{ml}$ of saline solution with the same injection rate. $30 \mathrm{~s}$ after the administration of contrast medium, dynamic MRM was performed with the same parameters and identical tuning conditions for totally 7 measurements. The contrastenhanced images were subsequently subtracted from the unenhanced images. On the subtraction images the region of interests (ROI's) were achieved on a pixel-by-pixel basis and signal-intensity curves were analysed. The kinetic behaviour of the lesion was analyzed by ROI in the most enhancing area. Also maximum intensity projections (MIP) were displayed.

Image interpretation

The MRM studies were interpreted by two examiners. One experienced and one trained observer classified the selected MR images anew, without knowledge of pathology. Neither was involved in rating the initial MR images, and both were blinded to the initial report and to the results of the histological and mammographic examinations. The rating was performed in consensus. In the case of different rating, the case was discussed until agreement was reached. All investigations were categorised according to BI-RADS: Mass/shape, mass/ margin, mass/enhancement, non-mass/distribution, non-mass/ internal enhancement, symmetry, kinetic curve assessment (24 missing) and T2-weighting (35 missing). These results were compared to histology. Statistical significance for the accuracy of MRM was calculated.

Reference standard

The authors chose the results of the histological examination as the reference standard for lesion evaluation. Findings could either be malignant or benign, and the type of tumour was recorded. All histological examinations were conducted by board-certified breast pathologists at the institute of pathology of our university hospital.

Data collection and statistical analysis

Data were recorded in a spreadsheet (Excel, Microsoft, Redmond, WA). Statistical analysis was performed with the chi-square and Fisher's exact tests using statistical software (SSPS, and MedCalc). According to the level of significance (two-sided p-value) asterisks were distributed:

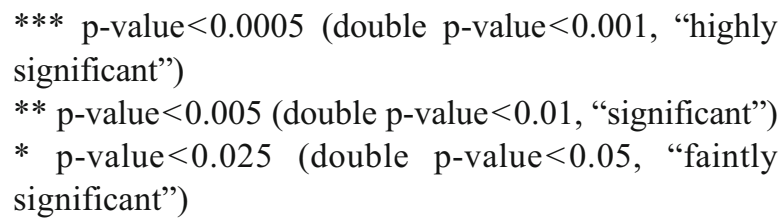

Table 4 Internal enhancement of the mass enhancement into histological proven malignant and benign tumors

\begin{tabular}{lllll}
\hline $\begin{array}{l}\text { Mass } \\
\text { Enhancement }\end{array}$ & $\begin{array}{l}\text { Malignant } \\
\text { Number (n) }\end{array}$ & $\begin{array}{l}\text { Benign } \\
\text { Number (n) }\end{array}$ & Total & p-value \\
\hline Rim enhancement & 43 & 1 & 44 & $0.0000^{* * *}$ \\
Heterogeneous & 35 & 23 & 58 & 0.1664 \\
Homogeneous & 1 & 8 & 9 & $0.0092^{*}$ \\
Heterogeneous + internal septation & 0 & 5 & 5 & $0.0192^{*}$ \\
\hline
\end{tabular}


Table 5 Distribution of the nonmass like-enhancement into histological proven malignant and benign tumors

\begin{tabular}{lllll}
\hline $\begin{array}{l}\text { Non-mass } \\
\text { Distribution }\end{array}$ & $\begin{array}{l}\text { Malignant } \\
\text { Number (n) }\end{array}$ & $\begin{array}{l}\text { Benign } \\
\text { Number (n) }\end{array}$ & Total & p-value \\
\hline Diffuse & 14 & 10 & 24 & 0.4133 \\
Multiple regions & 4 & 6 & 10 & 0.2761 \\
Regional & 5 & 14 & 19 & $0.0101^{*}$ \\
Ductal & 5 & 0 & 5 & 0.0445 \\
Segmental & 3 & 0 & 3 & 0.1564 \\
Dendritic & 1 & 0 & 1 & 0.5411 \\
Linear & 5 & 2 & 7 & 0.2958 \\
Focal & 0 & 3 & 3 & 0.0950 \\
\hline
\end{tabular}

\section{Results}

Comparison of benign and malignant tumours

The following tables show the morphological differences between malignant and benign tumours. For analyzing reasons we separated into mass and non-mass lesions. It was proven that $70.5 \%$ of the malignant tumours were categorised as mass, $23.2 \%$ as non-mass, and 7 malignant tumours were inconspicuous at MRM (6.3\%; not definitely detected in MRM, but with positive mammography or ultrasound investigation, and therefore with histological proven breast cancer). Out of the 95 benign tumours only $40 \%$ delivered a mass-diagnosis, $35.8 \%$, and $24.2 \%$ were considered as inconspicuous at MRM (Tables 3 and 4). Furthermore particular attention was paid on the histopathological diagnosis with regards to the specific BI-RADS criteria. The specific morphology is presented in the following tables.

Interpretation and reporting of MRM findings on the basis of the BI-RADS classification

\section{Focus}

In this matter it was not possible to give a significant statement, because only one malignant and one benign tumour showed a focal enhancement.
Mass

Shape The correlation of shape and diagnosis in mass enhancement is shown in Table 2 and gives the following results. 60 out of $68(88.2 \%)$ irregular formed masses are malignant. 11 out of $14(78.6 \%)$ oval shaped tumours have a benign histology. 14 of 25 (56\%) lobulated tumours were categorised as benign. Considering the group of 112 malignant tumours, $60(53.6 \%)$ out of them are formed irregularly.

Margin Correlation between margin and diagnosis in mass enhancement is given in Table 3. Most of the malignant lesions have an irregular $(42 \%)$ or spiculated $(25.9 \%)$ margin. Whereas $21.1 \%$ of benign lesions are of irregular margin, only $1.1 \%$ are spiculated. A smooth margin refers to benignity as 16 out 19 tumours of smooth margin were histological proven benign and only 3 were malignant.

Enhancement Table 4 shows that the majority of the malignant tumours had rim enhancement. The sheet points out, that 43 out of 44 tumours (97.7\%) with rim enhancement were categorised to the malignant group. Comparing the heterogeneous forms of enhancement, 35 tumours were into the malignant and 23 into the benign group. Homogeneous enhancement was seen in $88.9 \%(8 / 9$
Table 6 Internal enhancement of the non-mass-like enhancement in histological proven malignant and benign tumors

\begin{tabular}{lllll}
\hline $\begin{array}{l}\text { Non-mass } \\
\text { Central enhancement }\end{array}$ & $\begin{array}{l}\text { Malignant } \\
\text { Number (n) }\end{array}$ & $\begin{array}{l}\text { Benign } \\
\text { Number (n) }\end{array}$ & Total & p-value \\
\hline Heterogeneous & 28 & 17 & 45 & 0.1431 \\
Homogeneous & 1 & 1 & 2 & 0.7085 \\
Stippled & 5 & 12 & 17 & 0.0298 \\
Clumped & 2 & 3 & 5 & 0.4226 \\
Reticular/Dendritic & 1 & 3 & 4 & 0.2515 \\
\hline
\end{tabular}


Table 7 Symmetry for histological proven malignant and benign tumors

\begin{tabular}{|c|c|c|c|c|c|c|}
\hline \multirow{2}{*}{$\begin{array}{l}\text { Non-mass } \\
\text { Symmetry }\end{array}$} & \multicolumn{2}{|l|}{ Malignant } & \multicolumn{2}{|l|}{ Benign } & \multirow[t]{2}{*}{ Total } & \multirow[t]{2}{*}{ p-value } \\
\hline & Number (n) & $\%$ & Number (n) & $\%$ & & \\
\hline Symmetric & 1 & $0,9 \%$ & 9 & $9,5 \%$ & 10 & $0.0044 * *$ \\
\hline Asymmetric & 104 & $92,9 \%$ & 63 & $66,3 \%$ & 167 & $0.0000 * * *$ \\
\hline Inconspicuous & 7 & $6,3 \%$ & 23 & $24,2 \%$ & 30 & $0.0002 * * *$ \\
\hline Total & 112 & $100,0 \%$ & 95 & $100,0 \%$ & 207 & \\
\hline
\end{tabular}

cases) in tumours out of the benign group. Internal septation refers to benignity (Figs. 1-4).

\section{Non-mass}

Distribution There were only a few differences concerning non-mass between the two groups. However, all lesions with ductal and segmental distribution were categorised malignant. On the other hand, all focal lesions showed benign potential. For regional enhancement a mean significance for benignity was reported (Table 5, Fig. 3).

Internal enhancement 28 of 112 malignant tumours (25\%) had a heterogeneous central enhancement. Moreover, a stippled enhancement was predominantly seen in benign tumours (Table 6).

Symmetry Table 7 shows the malignant and benign lesions by means of their symmetry. The table indicates that the malignant as well as the benign tumours seemed to appear asymmetrically. Nevertheless, in 9 out of 10 cases symmetry leads highly significant to a benign tumour.

Kinetic Into the malignant group 69.6\% (78/112 tumours) of the lesions had a medium initial increase of the kinetic curve. Only $16.1 \%$ of the malignant tumours (18/112 cases) had a fast initial increase. 18 out of 20 tumours $(90 \%)$ with a fast kinetic were grouped as malignant (Table 8).

In the benign group $42.1 \%$ of the tumours (40/95 cases) showed a medium initial ascent of the curve. Out of the 16 tumours with slow initial increase 93.7\% (15 tumours) were highly significant categorised as benign (Table 8, Fig. 1).

The post initial increase of the signal for malignant and benign tumours is shown in Table 9. A plateau of the kinetic curve was seen in $70.5 \%$ (79/112 cases) into malignant lesions, and a wash-out was documented in $15.2 \%$ (17 cases). Out of a total number of 121 tumours with a plateau, 65.2\% (79 cases) were categorised as malignant. In addition $77.3 \%$ (17/22 cases) with wash-out were malignant. Conversely, a further increase of the kinetic curve was in $90 \%$ a marker for a benign tumour (Table 9).

T2-weighting It was noticeable that $77(69 \%)$ out of the malignant tumours had an intermediate T2-signal. This argued in $81.9 \%$ for malignancy. By comparison 37 of the benign tumours $(38.9 \%)$ had a hyperintense appearance (Table 10, Figs. 1, 2, 3 and 4).

\section{Discussion}

This study demonstrates a high value of a BI-RADS associated MRM algorithm to deliver a high accuracy for the diagnosis of breast tumours. The major finding of our study results which compared MRM findings with histology, is that on the basis of the BI-RADS classification an objective comparability and statement of diagnosis could be made highly significant.
Table 8 Initial kinetic curve assessment (Kinetic) for histological proven malignant and benign tumors

\begin{tabular}{lllll}
\hline Kinetic/Initial & $\begin{array}{l}\text { Malignant } \\
\text { Number (n) }\end{array}$ & $\begin{array}{l}\text { Benign } \\
\text { Number (n) }\end{array}$ & Total & p-value \\
\hline Slow & 1 & 15 & 16 & $0.0000^{* * *}$ \\
Mean & 78 & 40 & 118 & $0.0000^{* * *}$ \\
Fast & 18 & 2 & 20 & $0.0004^{* * *}$ \\
\hline
\end{tabular}




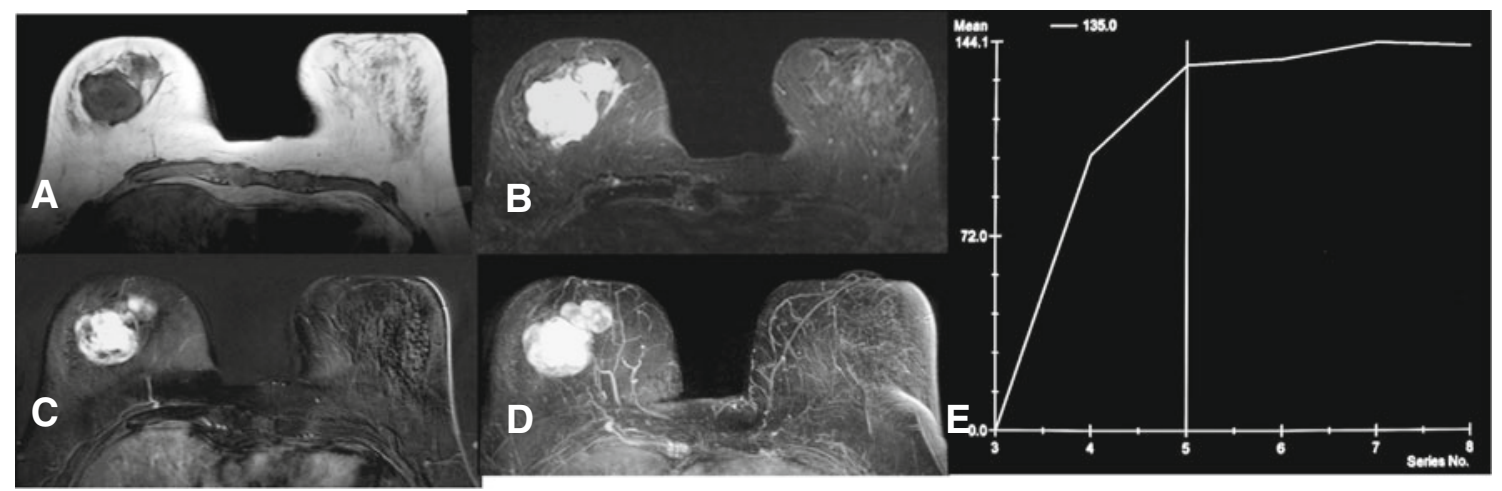

Fig. 1 Mass enhancement into the right breast (lobulated shape, irregular margin, heterogeneous enhancement) with hyperintensic T2-result. T1weighting (a), T2-weighting (b), subtraction (c), MIP-Reconstruction (d), Kinetic curve assessment (e). Histology: Phyllodes tumour

Gutierrez et al. evaluated the predictive features of BI-RADS lesion characteristics and the risk of malignancy for mammographic and clinical occult lesions detected initially on 1.523 MRMs. They concluded that combinations of BI-RADS lesion descriptors can predict the probability of malignancy for MRM masses but not for non-mass like enhancement [2]. In case of mass lesions, their results act in concert with our data. We showed as well, that specific BI-RADS criteria are strong and significant predictors for malignancy in unsuspected MRM mass lesions. Such confirmation led in about $65 \%$ of cases to a malignant diagnosis (Tables 2, 3 and 4). However, the combination of several marker and characteristics is useful to determine the likelihood of malignancy. In contrast to the results by Gutierrez et al. we could observe, that BI-RADS descriptions were as well predictive for malignancy of otherwise unsuspected MRM nonmass like lesions. Table 5 shows for example, that a nonmass like enhancement with ductal or segmental distribution was in $100 \%$ malignant.

In addition, our data showing particular BI-RADS lexicon descriptions to be strong predictors of malignancy, support recent work by Liberman et al. Their group investigated 427 lesions by MRM with dutcal enhancement. $80 \%$ out of the benign tumours had a plateau into the contrast-enhancement dynamic and $89 \%$ an isointensic signal into the T2-weighting [11]. Tozaki et al. described the PPV and NPV of focal masses. The main criterion for a malignant lesion was heterogeneous enhancement (96\%), while benign tumours showed inconspicuous margin and form in $82 \%$. The highest PPV for a malignant enhancement had lesions with spiculated margin $(100 \%)$, irregular form $(97 \%)$ and a heterogeneous enhancement without internal septation (97\%). The highest PPV for benignity was found in tumours with oval mass $(88 \%)$, homogeneous mass-enhancement (87\%) and heterogeneous massenhancement with internal septation (86\%). Combination of several specifications lead to a PPV of $100 \%$ for tumours with spiculated rim as well as for tumours with heterogeneous enhancement and post initial wash-out if those lesions were out of the group with oval margin [12].

However, non-mass like enhancement was identified as a challenging subgroup causing a high proportion of falsepositive diagnoses at diagnostic MRM. In several cases this lead to unnecessary biopsy [13]. In a further study by Tozaki et al. they investigated tumours without mass findings and PPVs were as well compared with each other. They concluded that most of the benign tumours were linear $(50 \%)$ and had a homogeneous internal pattern $(42 \%)$. In contrast, most of the malignant tumours were segmental (56\%), heterogeneous (44\%) and had a rim

Table 9 Post initial kinetic curve assessment (Kinetic) for histological proven malignant and benign tumors

\begin{tabular}{lllll}
\hline Kinetic/Post initial & $\begin{array}{l}\text { Malignant } \\
\text { Number (n) }\end{array}$ & $\begin{array}{l}\text { Benign } \\
\text { Number (n) }\end{array}$ & Total & p-value \\
\hline Plateau & 79 & 42 & 121 & $0.0000^{* * *}$ \\
Wash-out & 17 & 5 & 22 & $0.0170^{*}$ \\
Further increase & 1 & 9 & 10 & $0.0044^{* *}$ \\
\hline
\end{tabular}


Table 10 T2- weighting for histological proven malignant and benign tumors

\begin{tabular}{|c|c|c|c|c|c|c|}
\hline \multirow[b]{2}{*}{ T2-result } & \multicolumn{2}{|l|}{ Malignant } & \multicolumn{2}{|l|}{ Benign } & \multirow[t]{2}{*}{ Total } & \multirow[t]{2}{*}{ p-value } \\
\hline & Number (n) & $\%$ & Number (n) & $\%$ & & \\
\hline Hyperintensic & 11 & $9 \%$ & 37 & $38,9 \%$ & 48 & $0.0000 * * *$ \\
\hline Intermediate & 77 & $69 \%$ & 17 & $17,9 \%$ & 94 & $0.0000 * * *$ \\
\hline Inconspicuous & 7 & $6 \%$ & 23 & $24,2 \%$ & 30 & $0.0002 * * *$ \\
\hline Missing & 17 & $15 \%$ & 18 & $18,9 \%$ & 35 & 0.2958 \\
\hline Total & 112 & $100,0 \%$ & 95 & $100,0 \%$ & 207 & \\
\hline
\end{tabular}

Fig. 2 Non-mass like enhancement into the right breast (regional, heterogeneous enhancement), intermediate T2-result. T1- weighting (a), T2weighting (b), subtraction (c), MIP-Reconstruction (d). Histology: Invasive lobular carcinoma
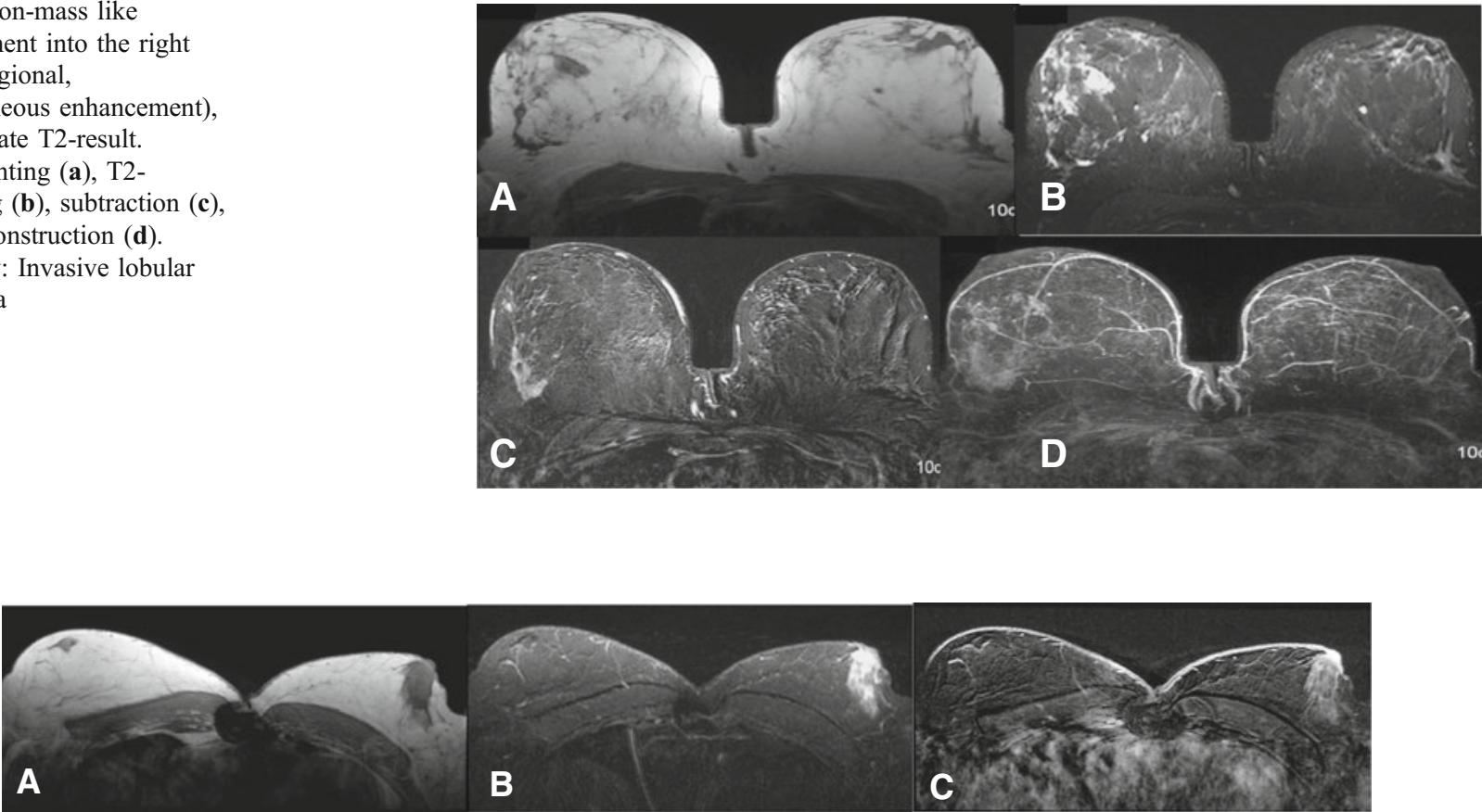

Fig. 3 Non-mass like enhancement into the left breast (regional, heterogeneous enhancement), hyperintensic T2-result. T1- weighting (a), T2weighting (b), Subtraction (c). Histology: Ductal hyperplasia

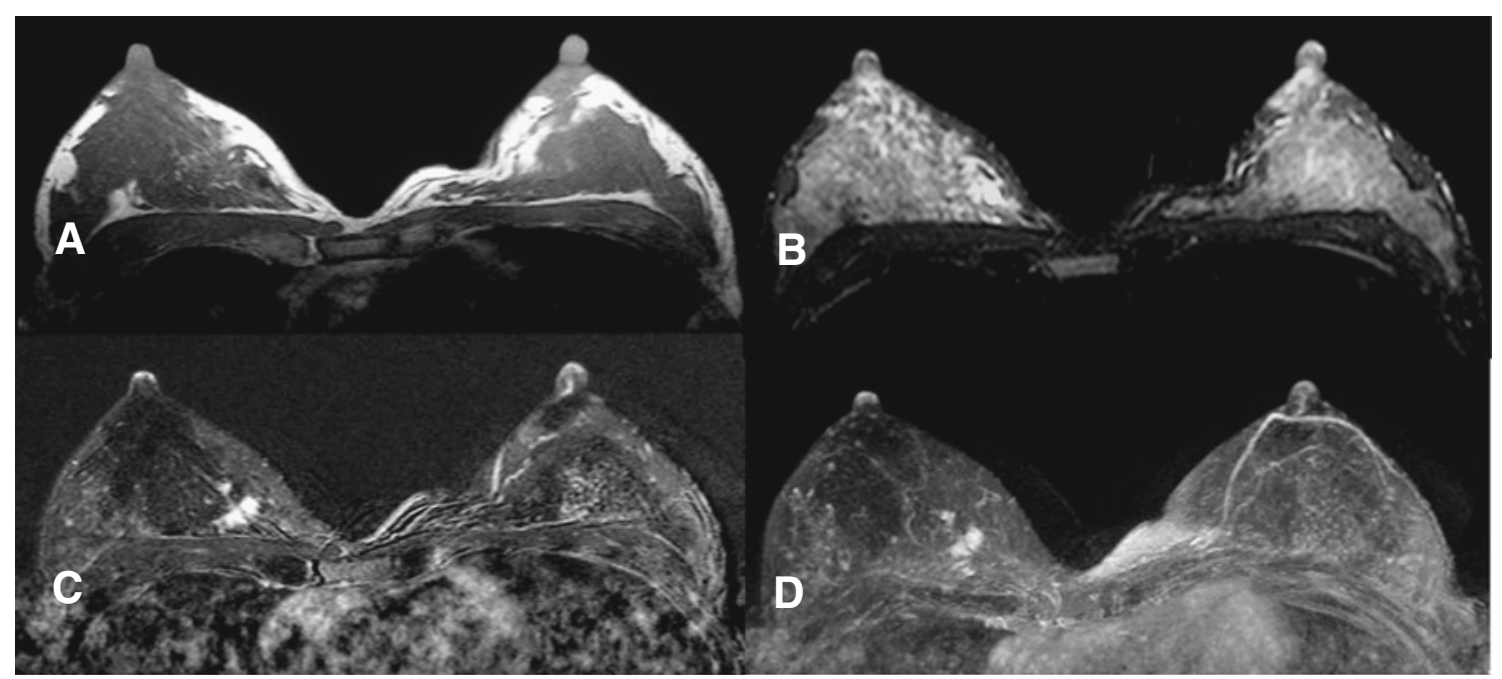

Fig. 4 Mass enhancement (irregular shape, irregular margin, heterogeneous) in the right breast with intermediate signal in T2. T1- weighting (a), T2- weighting (b), subtraction (c), MIP-Reconstruction (d). Histology: Radial scar 
enhancement. The highest PPV had carcinomas with a segmental distribution (100\%), rim enhancement (100\%) and grouped internal compaction (88\%) [14]. This acts in concert with our results. Mass enhancement (68\%) was significant for malignancy. This was confirmed by high significance (Table 2). A similar picture was shown for tumours without mass enhancement, $56.6 \%$ of them were benign. In this context, it has to be mentioned that $23 \%$ of the inconspicuous MR mammograms had malignant and $77 \%$ benign lesions (Table 2). Furthermore, our results indicated that $88 \%$ of the tumours with irregular shape were highly significant malignant. Those tumours with an oval or lobulated shape were in $78.6 \%$ (oval) and $56 \%$ (lobulated) benign. However, in these cases only a mean significance was reached. This leads to the conclusion that a lesion with an irregular shape is with the utmost probability malignant. In contrast, tumours with oval or lobulated shape suspect to be benign, without verifiable significance (Table 2). This is absolutely in line with former studies [11-14].

There was a strong tendency for tumours with irregular or spiculated margins to be malignant. Highly significant for benignity were tumours with smooth margin $(84.2 \%$; Table 3). If a lesion showed rim enhancement it was highly significant malignant (97.7\%). Tumours were significantly benign if there was a homogeneous enhancement (88.9\%) or a heterogeneous enhancement with internal septation (100\%; Table 4).

A non-mass like lesion with ductal or segmental distribution was in $100 \%$ malignant. Diffuse distribution was commonly seen within malignant tumours. However, it was not possible to constitute a distinct statistical statement (Table 5).

This corresponds to the study by Baltzer et al. which aimed to identify criteria for false-positive MRM findings in clinical practice. They concluded that non-mass like enhancement was the main cause for those findings. Furthermore, in their analysis the BI-RADS descriptions were not sufficient for differentiating malignant and benign non-mass like enhancement [15].

Evidence-based algorithms are needed to guide the radiologist through image assessment and to describe morphologic features. Thus, concerning the form of internal enhancement the range was essentially exceeded. 28 out of the malignant tumours showed heterogeneous enhancement, this lead to significance to malignancy only in $62 \%$. Marks for benignity were reticular (75\%) and stippled/punctuated enhancement $(70.58 \%)$. Whereas only $3.2 \%$ and $12.6 \%$ out of all benign tumours showed this pattern. Consequently, a significant statement could not be announced (Table 6). In addition, symmetry was very important to deliver a prognosis. $90 \%$ out of the symmetric appeared enhancement $(9.5 \%$ of the benign tumours) were significantly benign. Asymmetric appeared enhancement was highly significant for malignancy (Table 7).

A number of investigations have been conducted to introduce and describe the kinetic features of breast lesions $[12,13]$. Tumours with a mean or fast signal increase had a high significance to be malignant in $66 \%$ or $90 \%$. It was well-defined, that tumours with a slow signal increase were highly significant benign (in 94\%). Furthermore, it was shown that lesions with a plateau or a wash-out were significant malignant. Only $44 \%$ of the benign group showed a plateau (Tables 8 and 9). Within the T2weighting a strong tendency was seen, that tumours with an intermediate signal were in $82 \%$ highly significant malignant. On the other hand, a hyperintense signal in the T2- weighting was in $77 \%$ an indicator for benignity (Table 10).

Finally we conclude, based on the BI-RADS classification, that an objective comparability and highly significant statement of diagnosis can be made $[2-6,12-16]$. The combination of several marker and characteristics is still essential to determine malignancy. Due to numerous false-negative and false-positive MRM-results histology is still essential.

Open Access This article is distributed under the terms of the Creative Commons Attribution Noncommercial License which permits any noncommercial use, distribution, and reproduction in any medium, provided the original author(s) and source are credited.

\section{References}

1. Aubard Y, Genet D, Eyraud JL et al (2002) Impact of screening on breast cancer detection. Retrospective comparative study of two periods ten years apart. Eur J Gynaecol Oncol 23:37-41

2. Gutierrez RL, DeMartini WB, Eby PR et al (2009) BI-RADS lesion characteristics predict likelihood of malignancy in breast MRI for masses but not for non mass like enhancement. AJR 193:994-1000

3. Nunes LW, Schnall MD, Orel SG et al (1997) Breast MR imaging: interpretation model. Radiology 202:833-841

4. Nunes LW, Schnall MD, Orel SG (2001) Update of breast MR imaging architectural interpretation model. Radiology 219:484-494

5. Schnall MD, Blume J, Bluemke DA et al (2006) Diagnostic architectural and dynamic features at breast MR imaging: multicenter study. Radiology 238:42-53

6. Benndorf M, Baltzer PA, Vag T et al (2010) Breast MRI as an adjunct to mammography: Does it really suffer from low specificity? A retrospective analysis stratified by mammographic BI-RADS classes. Acta Radiol 51:715-721

7. Obenauer S, Sohns C, Werner C et al (2006) Computer-aided detection in full-field digital mammography: detection in dependence of the BI-RADS categories. Breast J 12:16-19

8. American College of Radiology: Breast Imaging Reporting and Data System (BI-RADS). 3. Auflage, Reston 1998 
9. Schnall MD, Ikeda DM (1999) Lesion diagnosis working group on breast MR. Radiology 153:243-244

10. Ikeda DM, Hylton MN, Kinkel K et al (2001) Development, standardization, and testing of a lexicon for reporting contrastenhanced breast magnetic resonance imaging studies. J Magn Reson Imaging 13:889-95

11. Liberman L, Morris EA, Dershaw DD, Abramson AF, Tan LK et al (2003) Ductal enhancement on MR imaging of the breast. AJR 181:519-525

12. Tozaki M, Fukuda K (2006) High-spatial-resolution MRT of nonmass like breast lesions: interpretation model based on BI-RADS MRT descriptors. AJR 187:330-337
13. Kuhl CK (2007) Current status of breast MR imaging. Part 2. Clinical applications. Radiology 244:672-691

14. Tozaki M, Igarashi T, Fukuda K (2006) Positive and negative predictive values of BI-RADS-MRT, descriptors for focal breast masses. Magn Reson Med Sci 5:7-15

15. Baltzer PAT, Benndorf M, Dietzel M et al (2010) False-positive findings at contrast-enhanced breast MRI: a BI-RADS descriptory study. AJR 194:1658-1663

16. Tozaki M, Igarashi T, Fukuda K (2006) Breast MRT using the VIBE sequence: clustered ring enhancement in the differential diagnosis of lesions showing non-masslike enhancement. AJR $187: 313-321$ 